\title{
¿Existen diferencias en función del sexo en comportamientos socialmente
}

\author{
responsables en universitarios?
}

\section{Are there differences in students' ${ }^{\prime}$ responsible social behaviour according to gender role?}

\author{
Ana María Morales Rodríguez*, Francisco Manuel Morales Rodríguez*, Manuel Alejandro Narváez Peláez * \\ *Universidad de Málaga
}

\begin{abstract}
Resumen
El presente trabajo aporta una evaluación de comportamientos socialmente responsables en una muestra de 53 estudiantes universitarios de posgrado entre 24 y de 49 años de edad del Máster en Profesorado de Educación Secundaria de la Facultad de Ciencias de la Educación de la Universidad de Málaga analizando diferencias en función del sexo. Con respecto a los resultados se encuentra que hay diferencias estadísticamente significativas en función del sexo en la frecuencia en la que tiene lugar los comportamientos socialmente responsables en esta muestra de futuros docentes.
\end{abstract}

Palabras clave: competencias solidarias, sexo, comportamiento socialmente responsable.

Abstract

This work contributes to a socially responsible behaviours assessment, analysing the differences between gender. It is based on a sample of 53 postgraduate students, aged 24 to 29, of "Aprendizaje y Desarrollo de la Personalidad" (Learning and Personality Development), from the master's degree in "Educación Secundaria (junior high school) Teaching" at Educational Sciences Faculty, University of Málaga. Regarding the results, there are some statistically significant differences according to the gender, and how frequent the socially responsible behaviours take place.

Keywords: supportive competences, gender, socially responsible behaviour

El presente trabajo se enmarca en un Proyecto de Innovación Educativa (PIE13-031) en el que se pretende ampliar y consolidar el uso herramientas que permitan la evaluación formativa de competencias transversales en la docencia práctica en las ramas de conocimiento de Ciencias y Ciencias de la Salud; así como proyectar también su aplicación en las ramas de Ciencias Sociales y Jurídicas y en la docencia Internacional.

Considerándose en dicho proyecto que la excelencia del EEES supone el aprendizaje basado en competencias y un cambio paradigmático en la enseñanza universitaria española, una de las competencias transversales para fomentar son las llamadas competencias interpersonales dentro de las que pueden encontrarse actitudes, valores y comportamientos ética y socialmente responsables.

El presente trabajo aporta una evaluación de comportamientos socialmente responsables en una muestra de 53 estudiantes universitarios de posgrado analizando diferencias en función del sexo.

Estudios previos existentes que analizan valores y actitudes socialmente responsables en función del sexo encuentran en las mujeres mayor puntuación en actitudes y comportamiento socialmente responsables en comparación con los hombres (Arango-Tobon, Clavijo-Zapata, Puerta-Lopera y Sanchez-Duque, 2014; Navarro et al., 2012) y mayor preocupación por los demás (Arango et al., 2014).

Otros trabajos (Redondo y Guevara, 2012) evidencian mayor comportamiento prosocial en las mujeres así como una mayor predisposición empática (Galán, Romero, Morillo y Alarcón, 2014), en comportamientos relacionados con la "sociabilidad y solidaridad para la convivencia" (Navarro et al., 2012) y con la llamada "ética de cuidado" (Gilligan, 1992).

En nuestro contexto se hace necesario seguir profundizando en el papel de la variable sexo en la generación de diferencias en la autoatribución de comportamientos socialmente responsables.

\section{Método}

\section{Participantes}

Los participantes han sido 53 estudiantes universitarios de posgrado entre 24 y de 49 años de edad del Máster en Profesorado de Educación Secundaria de la Facultad de Ciencias de la Educación de la Universidad de Málaga.

\section{Instrumentos o Materiales}

Se ha utilizado para evaluar los comportamientos socialmente responsable el siguiente instrumento:

Cuestionario de autoatribución de comportamientos socialmente responsables en universitarios (CACSR, Davidovich, Espina, Navarro y Salazar, 2005). Este instrumento incluye dos escalas: una de frecuencia y otra de intencionalidad. Está compuesto por un total de 40 ítems según escala tipo Likert de cinco escalones según se trate de la escala de frecuencia $(1=$ Nunca; $2=$ Casi nunca; $3=$ A veces; $4=$ Casi siempre y $5=$ Siempre) o la escala de intención ( $1=$ No aplica; 2 = Beneficio personal; $3=$ No tengo clara la intención; 4= Beneficio para otros y $5=$ Beneficio mutuo). Ambas escalas están formadas por las siguientes diez categorías: Responsabilidad laboral/académica, actividades de voluntariado, ayuda social, actividades religiosas, convivencia social, responsabilidad cívica, autocuidado, desarrollo cultural, ecología y medio ambiente, y respeto por espacios compartidos.

Este instrumento es útil para evaluar la frecuencia de autoatribución de comportamiento socialmente responsable. 


\section{Procedimientos}

Se ha insistido en la privacidad y en el carácter voluntario de la participación sin particularizar entre grupos o personas en el tratamiento de los datos. El CACSR se aplicó de forma colectiva dentro del horario académico establecido en el centro y fue administrado por un investigador. Para analizar las diferencias en comportamientos sociales en función del sexo se aplicó la $t$-Student para muestras independientes.

\section{Resultados}

Con respecto a los resultados se encuentra que hay diferencias estadísticamente significativas en función del sexo en la frecuencia en la que tiene lugar los comportamientos socialmente responsables en esta muestra de futuros docentes.

Es destacable que los hombres de esta muestra participan en mayor medida que las mujeres en campañas de donación de sangre.

En cambio, las mujeres colaboran más en la formación de otras personas (ayuda a niños y niñas con problemas de aprendizaje; enseñanza lectoescritura a adultos; ayudas en el estudio a otros compañeros y compañeras, etc); compran en mayor medida productos en los que un porcentaje de su utilidad se destina a fundaciones y asociaciones de ayuda social y trabajo a favor de otros.

Las mujeres también afirman entregar en mayor medida que los hombres recursos materiales a campañas de ayuda social (alimentos, ropa y otros materiales).

A continuación se muestran en la Tabla 1 los resultados estadísticamente significativos más relevantes; presentándose las medias y desviaciones típicas así como los resultados de la diferencias de medias en comportamientos socialmente responsables en función del sexo.

Tabla 1

Diferencia de medias en comportamiento socialmente responsable en función del sexo

\begin{tabular}{|c|c|c|c|}
\hline $\begin{array}{l}\text { Comportamiento } \\
\text { socialmente } \\
\text { responsable }\end{array}$ & $\begin{array}{l}\text { Hombres } \\
\text { Media } \\
\text { (dt) }\end{array}$ & $\begin{array}{l}\text { Mujeres } \\
\text { Media } \\
(d t)\end{array}$ & $\begin{array}{l}g l=51 \\
t\end{array}$ \\
\hline $\begin{array}{l}\text { 1.Participo en } \\
\text { campañas de } \\
\text { donación de sangre }\end{array}$ & $\begin{array}{l}2.81 \\
(1.23)\end{array}$ & $\begin{array}{l}1.95 \\
(1.19)\end{array}$ & $2.51 * *$ \\
\hline $\begin{array}{l}\text { 2.Colaboro en la } \\
\text { formación de otras } \\
\text { personas }\end{array}$ & $\begin{array}{l}3.42 \\
(1.14)\end{array}$ & $\begin{array}{l}4.15 \\
(.93)\end{array}$ & $-2.38 *$ \\
\hline $\begin{array}{l}\text { 3.Compro productos } \\
\text { que entregan un } \\
\text { porcentaje de su } \\
\text { utilidad a fundaciones } \\
\text { de ayuda social } \\
\text { (comercio justo) }\end{array}$ & $\begin{array}{c}2.45 \\
(.83)\end{array}$ & $\begin{array}{l}2.95 \\
(.75)\end{array}$ & $-2.16^{*}$ \\
\hline $\begin{array}{l}\text { 4.Entrego recursos } \\
\text { materiales a campañas } \\
\text { de ayuda social, tales } \\
\text { como: vestimentas, } \\
\text { alimentos, materiales } \\
\text { de construcción, etc. }\end{array}$ & $\begin{array}{l}3.09 \\
(1.01)\end{array}$ & $\begin{array}{l}3.85 \\
(1.22)\end{array}$ & $-2.44 * *$ \\
\hline
\end{tabular}

Significación estadística. ${ }^{*} p<.05 ; * *$

\section{Discusión y Conclusiones}

Los resultados demuestran que, en esta muestra de universitarios, existen diferencias en la autoatribución de comportamientos socialmente responsables en función del sexo.

Concretamente, son las mujeres las que manifiestan estar comprometidas con un mayor número de comportamientos responsables socialmente; resultado que también coincide con investigaciones previas en las que el sexo produce diferencias en la atribución de comportamiento socialmente responsables a favor de las mujeres (Arango-Tobon et al., 2014; Bustamante y Navarro, 2009).

Los hombres muestran mayor puntuación media en el comportamiento "Participo en campañas de donación de sangre". Este resultado es congruente con otro estudio (Vásquez, Ibarra y Maldonado, 2007) en población universitaria en el que se encuentra en los hombres mayor predisposición a donar sangre en comparación con las mujeres; aunque hay que señalar que dichas diferencias en la investigación de Vásquez et al. (2007) no llegan a ser estadísticamente significativas.

Se concluye señalando la utilidad de estos datos para continuar con la formación, enseñanza y aprendizaje de este tipo de competencias sociales y ciudadanas considerando la importancia de variables mediadoras como el sexo.

Este trabajo es susceptible de mejora. Puede ampliarse la muestra y se pueden realizar diseños multivariados y longitudinales en los que se consideren otras variables como el curso y el estatus socioeconómico. No obstante, como se ha puesto de manifiesto, proporciona información útil en nuestro contexto que genera toma de conciencia sobre la importancia de realizar intervenciones educativas diferentes considerando la importancia y efectos de variables como el sexo.

\section{Referencias}

Arango-Tobon, O. E., Clavijo-Zapata, S. J., Puerta-Lopera, I. C. y Sanchez-Duque, J. W. (2014). Formación académica, valores, empatía y comportamientos socialmente responsables en estudiantes universitarios. Revista de la Educación Superior, 43(169), 89-105. Recuperado de: http://www.redalyc.org/articulo.oa?id=60430753006

Bustamante, M. y Navarro, G. (2009). Autoatribución de comportamientos socialmente responsables en estudiantes de carreras del área de Ciencias Sociales. Revista Perspectiva, 18, 45-121. Recuperado de: http://dialnet.unirioja.es/descarga/articulo/2573403. pdf

. Davidovich, M. P., Espina, A., Navarro, G. y Salazar, L. (2005). Construcción y estudio piloto de un cuestionario para evaluar comportamiento socialmente responsables en estudiantes universitarios. Revista de Psicología de la Universidad de Chile, 14(1),125-139.

Galán, J. M., Romero, M., Morillo, R. y Alarcón, J. M. (2014). Descenso de empatía en estudiantes de enfermería y análisis de posibles factores implicados. 
Psicología Educativa, 20, 53-60. doi: 10.1016/j.pse. 2014.05.007

Gilligan, C. (1992). Uma voz diferente: Psicologia da diferença entre homens e mulheres da infância a idade adulta. Rio de Janeiro: Rosa dos Tempos.

Navarro, G., Boero, P., Jiménez, G., Tapia, L., Hollander, R., Escobar, A., ... Espina, A. (2012). Universitarios y Responsabilidad Social. Calidad en la Educación, 36, 123-147.
Redondo, J. y Guevara, E. (2012). Diferencias de género en la prevalencia de la conducta prosocial y agresiva en adolescentes de dos colegios de la ciudad de Pasto. Revista Virtual Universidad Católica del Norte, 36, 173-192.

Vásquez, M., Ibarra, P., y Maldonado, M. (2007). Conocimientos y actitudes hacia la donación de sangre en una población universitaria de Chile. Revista Panamericana de Salud Pública, 22(5), 323-328. 